\title{
High occurrence of thyroid multinodularity and low occurrence of subclinical hypothyroidism among tobacco smokers in a large population study
}

\author{
N Knudsen ${ }^{1,2}$, I Bülow ${ }^{3}$, P Laurberg ${ }^{3}$, H Perrild ${ }^{1}$, L Ovesen ${ }^{4}$ \\ and $\mathbf{T}$ Jørgensen ${ }^{2}$ \\ ${ }^{1}$ Department of Internal Medicine I, Bispebjerg Hospital, University of Copenhagen, Copenhagen, Denmark \\ ${ }^{2}$ Centre for Preventive Medicine, Glostrup Hospital, University of Copenhagen, Copenhagen, Denmark \\ ${ }^{3}$ Department of Endocrinology, Aalborg Hospital, Aalborg, Denmark \\ ${ }^{4}$ Institute of Food Research and Nutrition, The Danish Veterinary and Food Administration, Soeborg, Denmark \\ (Requests for offprints should be addressed to N Knudsen, Erantishaven 108, DK-2765 Smoerum, Denmark; Email: nk01@bbh.hosp.dk)
}

\begin{abstract}
Tobacco smoking increases the risk of goitre and Graves' disease, but the association with thyroid nodularity and hypothyroidism has not been settled. We investigated 4649 subjects from the general population with questionnaires, thyroid ultrasonography and blood tests. The results were analysed in multivariate regression models. Tobacco smoking was associated with an increased prevalence of thyroid multinodularity (odds ratio (OR) 1.9; 95\% confidence interval (CI) $1 \cdot 4-2 \cdot 5)$, but not with increased prevalence of solitary thyroid nodules. The tendency was for a stronger association in the area with the most pronounced iodine deficiency $(P$ for interaction $=0 \cdot 08)$.
\end{abstract}

Lower levels of serum TSH were found among tobacco smokers $(P<0 \cdot 001)$, but this association disappeared when adjustment was made for thyroid nodularity and thyroid volume. The prevalence of elevated TSH levels was markedly reduced among smokers (OR 0.47; 95\% CI $0 \cdot 33-0 \cdot 67)$. No association was found between smoking and hyperthyroidism. The observed associations seem to be explainable by the blocking of iodine uptake and organification in the thyroid by thiocyanate, a degradation product of cyanide in tobacco smoke.

Journal of Endocrinology (2002) 175, 571-576

\section{Introduction}

A higher goitre prevalence and higher thyroid volumes are found among tobacco smokers than among non-smokers in most studies from iodine-deficient areas (Hegedus et al. 1985, Nygaard et al. 1993, Georgiadis et al. 1997, Barrere et al. 2000, Brix et al. 2000, Knudsen et al. 2002), whereas the association is weaker in iodine-sufficient areas. This has lead to the suggestion of a synergistic effect of iodine deficiency and tobacco smoking on the thyroid, which is in line with experimental studies suggesting that the goitrogenic effect of tobacco smoke is due to thiocyanate acting as a competitive inhibitor of iodine uptake and organification in the thyroid (Fukayama et al. 1992).

Iodine deficiency is regarded as a risk factor for thyroid nodules, though results from epidemiological studies are ambiguous. As smoking is probably mimicking iodine deficiency, increased prevalence of thyroid multinodularity and multinodular toxic goitre among smokers could be anticipated, as has been observed in iodine-deficient areas (Laurberg et al. 1991).
In addition, increased incidences of Graves' disease and especially ophthalmopathy have been observed among smokers (Bartalena et al. 1995). This is probably due to modification of the autoimmune responses among smokers (Bartalena et al. 1995). Generally, lower levels of serum thyrotrophin (TSH) have been found among smokers in most studies (Bertelsen \& Hegedus 1994), an association that cannot be explained by the increased number of Graves' patients. The lower serum TSH levels could be due to increased evolution of thyroid autonomy caused by iodine depletion of the thyroid by thiocyanate; however, sympathetic nervous stimulation (Melander et al. 1977), induction of liver enzymes (Hart et al. 1976), or inhibition of TSH secretion (Bartalena et al. 1995) could also be involved. A recent, Danish case-control study found increased occurrence of hypothyroidism among smokers, but this association is controversial (Vestergaard et al. 2002).

We report data from a recent, large, cross-sectional study with registration of thyroid nodules with ultrasonography after validated principles and, furthermore, 
thyroid function was registered. The association with smoking habits was analysed and the possible synergy between tobacco smoking and iodine deficiency was evaluated.

\section{Subjects and Methods}

A cohort of 9274 subjects was randomly selected from the Danish Civil Registration System within certain age groups. The age groups were chosen to represent women before childbearing age (18-22 years), women within childbearing age (25-30 years), premenopausal women after childbearing age (40-45 years), postmenopausal women (60-65 years), and a group of men for gender comparisons in the age spectrum with the expected highest prevalence of thyroid abnormalities (60-65 years). The cohort was sampled in two Danish cities: Aalborg from the western part of Denmark representing moderate iodine deficiency ( $45 \mu \mathrm{g}$ iodine/1 in spot urine samples), and Copenhagen from the eastern part of Denmark representing mild iodine deficiency $(61 \mu \mathrm{g} / \mathrm{l})$. For the classification of iodine status, individuals taking iodine supplementation $(n=1310)$ were not included. The participation rate was $50 \cdot 1 \%$, leaving 4649 subjects for the analyses. The cohort has previously been described in detail (Knudsen et al. 2000a).

Thyroid nodularity was determined by ultrasonography after standardized criteria as previously described (Knudsen et al. 1999a), and the classification according to thyroid nodularity has been found to be reproducible between observers (Knudsen et al. 1999a). Thyroid nodules were registered from a diameter of $5 \mathrm{~mm}$ but, for the analyses in this paper, only nodules $\geq 10 \mathrm{~mm}$ in diameter were included to ensure the clinical relevance of the nodules and to reduce misclassification. The structure of thyroid glands was categorized as normal, as multinodular, or as containing a solitary nodule.

Serum TSH, free tri-iodothyronine (T3) and free thyroxine (T4) were analysed with immunoluminometric assays (LUMItest; BRAHMS, Berlin, Germany). The functional sensitivity of the TSH assay was $0 \cdot 01 \mathrm{mU} / 1$. Participants were divided into three groups according to thyroid function: normal (serum TSH $0.4-3.6 \mathrm{mU} / \mathrm{l}$ ), hypothyroid (serum $\mathrm{TSH}>3.6 \mathrm{mU} / \mathrm{l}$ ) or hyperthyroid (serum $\mathrm{TSH}<0 \cdot 4 \mathrm{mU} / \mathrm{l}$ ). Only thyroid dysfunction according to TSH values was used (subclinical thyroid dysfunction), as the number of participants with overt thyroid dysfunction was too small to allow reliable analyses. The limits for TSH corresponded to the $2 \cdot 5$ th and 97.5th percentiles among subjects with no known thyroid disease and normal ultrasonography of the thyroid (Knudsen et al. 2000b). Thyroid peroxidase (TPO) antibodies were analysed with a radioimmunoassay (DYNOtest; BRAHMS), and autoimmune hypothyroidism was defined as serum TPO antibodies $>60 \mathrm{kU} / 1$ (manufacturer's cut-off) and serum $\mathrm{TSH}>3.6 \mathrm{mU} / 1$. No follow-up on abnormal thyroid function tests was performed; participants with biochemical signs of overt thyroid dysfunction were advised to consult their general physician for checking.

Tobacco smoking habits were registered from questionnaires and subsequent interviews with a physician. Participants were asked about present or previous smoking, daily or occasional smoking, amount of tobacco consumed and type of smoking (cigarettes, cheroots, cigars or pipe tobacco). To combine the different types of tobacco, cigarettes were regarded as $1 \mathrm{~g}$ tobacco, cheroots $2 \mathrm{~g}$, and cigars $5 \mathrm{~g}$; the participants were stratified according to gram tobacco consumed corresponding to number of cigarettes. Smokers were classified as non-smokers, ex-smokers, moderate smokers (1-19 g/day) and heavy smokers (minimum $20 \mathrm{~g} /$ day), or simply as present smokers or non-smokers dependent on the context.

To account for possible confounding of the analyses, alcohol consumption, familial occurrence of thyroid disease and iodine excretion in the four groups were also registered.

\section{Statistics}

Data processing was done with the statistical software SPSS, version $10 \cdot 0$, Scandinavian edition (SPSS, Holte, Denmark). To allow for adjustment for possible confounding in the analyses, all dichotomous variables (multinodularity, solitary nodule, hypo- and hyperthyroidism) were analysed in logistic regression analyses, and continuous variables (serum TSH, free T3 and free T4) were analysed in linear models. Serum TSH was analysed after logarithmic transformation and transformed back before presentation, as the distribution was skewed towards higher values, but normally distributed after transformation.

Age, gender and region of inhabitancy (and thereby iodine status) were included in all regression models, as smoking habits were unevenly distributed between groups and, furthermore, the prevalence of thyroid nodularity as well as thyroid function varied between groups. Familial occurrence of thyroid disease, alcohol consumption and iodine excretion in casual urine samples were tested in all models as confounders, but had no impact on the estimates for the association between tobacco smoking and thyroid disease, and they were not included in the final models. First-order interactions with smoking were tested in all models, but they were not found to be significant. One interaction term has been included in the presentation of data, however, as a trend towards interaction was found between iodine status and smoking in the association with thyroid multinodularity, and this interaction could be important for the interpretation of data.

Tobacco smoking was evaluated as a continuous variable as well as in groups. Only minor differences were 
Table 1 The distribution of the 4649 participants in the population study on some central variables

\begin{tabular}{|c|c|c|}
\hline & Parameter & $\begin{array}{l}\text { Number of } \\
\text { participants }\end{array}$ \\
\hline \multicolumn{3}{|l|}{ Variable } \\
\hline \multirow[t]{2}{*}{ Eligibility } & Known thyroid disease & 228 \\
\hline & Eligible & 4421 \\
\hline \multirow[t]{2}{*}{ lodine status } & Mild iodine deficiency & 2320 \\
\hline & Moderate iodine deficiency & 2101 \\
\hline \multirow[t]{4}{*}{ Thyroid structure } & No nodules & 3656 \\
\hline & Solitary nodule & 262 \\
\hline & Multiple nodules & 497 \\
\hline & Missing value & 6 \\
\hline \multirow[t]{4}{*}{ Thyroid function } & $\begin{array}{l}\text { Normal } \\
(\text { TSH } 0 \cdot 4-3 \cdot 6 \mathrm{mU} / \mathrm{l})\end{array}$ & 3959 \\
\hline & $\begin{array}{l}\text { Hyperthyroid } \\
(\mathrm{TSH}<0 \cdot 4 \mathrm{mU} / \mathrm{l})\end{array}$ & 214 \\
\hline & $\begin{array}{l}\text { Hypothyroid } \\
(\mathrm{TSH}>3.6 \mathrm{mU} / \mathrm{l})\end{array}$ & 188 \\
\hline & Missing value & 60 \\
\hline \multirow[t]{5}{*}{ Tobacco smoking } & Never smoked & 1817 \\
\hline & Ex-smokers & 983 \\
\hline & Moderate smokers & 1037 \\
\hline & Heavy smokers & 582 \\
\hline & Missing value & 2 \\
\hline
\end{tabular}

found between the continuous variable and a grouped variable and, finally, a variable with four groups was chosen as appropriate regarding the association with thyroid nodularity. On the contrary, four groups were found to be no more informative than two with regard to the association with thyroid function, as small and insignificant differences were found between non-smokers and ex-smokers, and between moderate and heavy smokers.

\section{Results}

All participants with known thyroid disease prior to this investigation were excluded from these analyses. The number of subjects eligible for each analysis is summarized in Table 1.

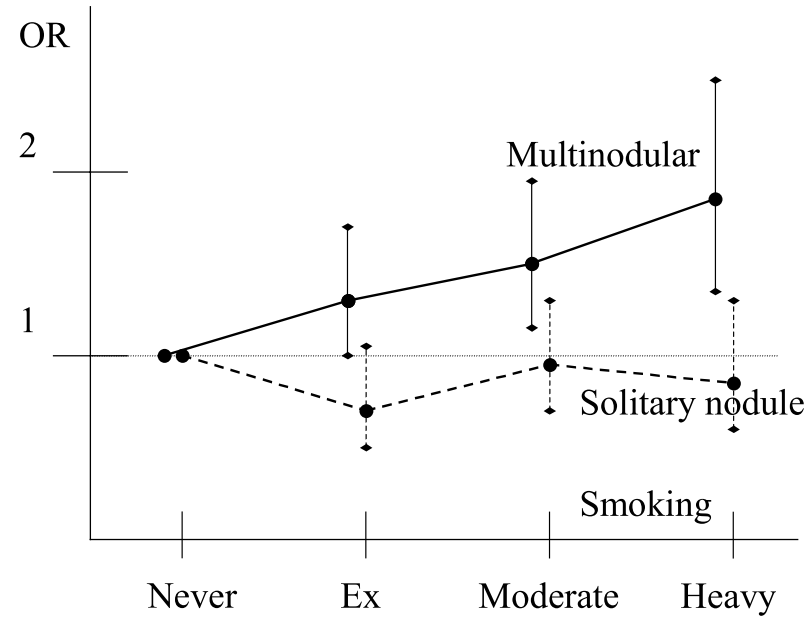

Figure 1 The association between tobacco smoking and multiple nodules or a solitary nodule in the thyroid at an ultrasonographic investigation of 4412 unselected Danes with no previous thyroid disease. Vertical bars represent 95\% Cl. OR values are significantly different from those who have never smoked if the $\mathrm{Cl}$ does not include 1.

A positive association was found between tobacco smoking and the occurrence of multiple nodules in the thyroid at ultrasonography. The observed prevalence of multinodularity was $7 \cdot 6 \%$ among non-smokers and $16 \cdot 5 \%$ among heavy smokers. The difference was slightly less when adjusted for age, gender and iodine status in a multivariate model as reflected by an odds ratio (OR) of 1.9 (95\% confidence interval (CI) 1.4-2.5) (Fig. 1). On the other hand, no association or any trend towards an association was found between tobacco smoking and the occurrence of solitary thyroid nodules (Fig. 1).

The association between tobacco smoking and thyroid multinodularity seemed different in the two regions with different iodine status, as apparently the association was stronger in the area with the most pronounced iodine deficiency (Fig. 2). The difference in the association was not statistically significant, however $(P$ for interaction $=0 \cdot 08)$.

Serum TSH was significantly lower among smokers than among non-smokers and correspondingly higher

Table 2 The association between smoking habits and thyroid hormone levels in a Danish population study. Values are means with $95 \% \mathrm{Cl}$ (logarithmic transformation was used for TSH in the analyses) in a linear model correcting for age, sex and iodine status of region of inhabitancy; 4361 participants were eligible for these analyses

\begin{tabular}{|c|c|c|c|}
\hline & Smokers & Non-smokers & $P$ \\
\hline Serum TSH & $1 \cdot 14(1 \cdot 11-1 \cdot 19)$ & $1 \cdot 28(1 \cdot 24-1 \cdot 32)$ & $<0 \cdot 001$ \\
\hline Serum free $\mathrm{T} 3$ & $5 \cdot 16(5 \cdot 10-5 \cdot 22)$ & $5 \cdot 20(5 \cdot 16-5 \cdot 24)$ & $0 \cdot 27$ \\
\hline Serum free $\mathrm{T} 4$ & $14.94(14.79-15 \cdot 09)$ & $14 \cdot 69(14 \cdot 58-14 \cdot 80)$ & $0 \cdot 008$ \\
\hline
\end{tabular}

The associaiton became statistically insignificant when adjustment for thyroid nodularity and thyroid volume was made. 


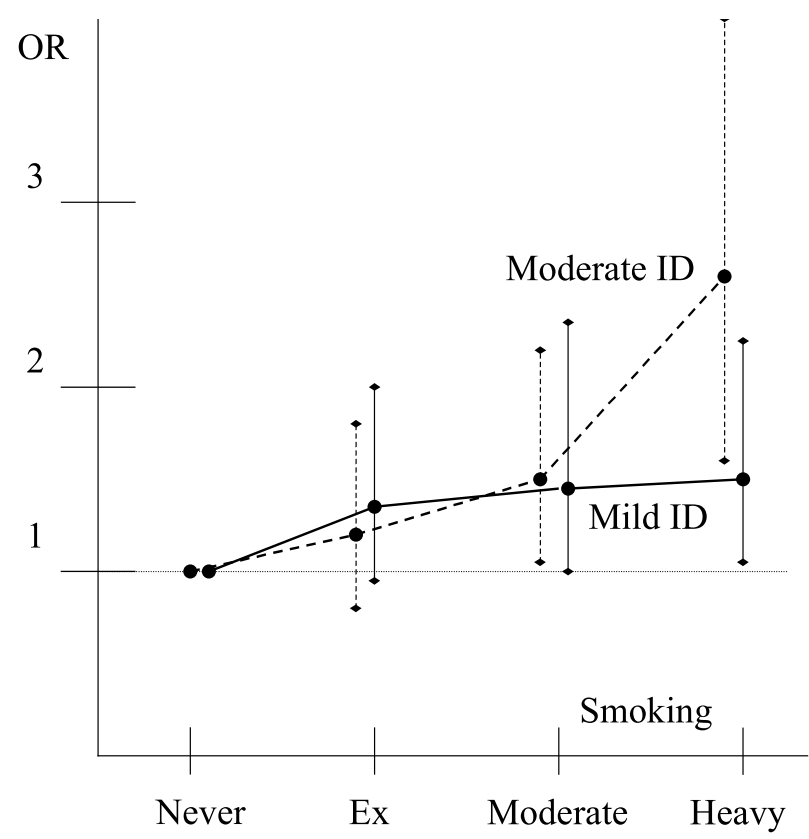

Figure 2 The association between smoking and multinodular appearance of the thyroid gland at ultrasonography. Data from a logistic regression analysis including 4412 unselected Danes without previous thyroid disease from two regions with slightly different degrees of iodine deficiency (ID). OR values are adjusted for age and gender. Vertical bars represent $95 \% \mathrm{Cl}$. OR values are significantly different from those who have never smoked if the $\mathrm{Cl}$ does not include 1 .

levels of serum free T4 were found among smokers (Table 2). These differences disappeared completely if adjustment was made for thyroid volume and thyroid nodularity in the model, suggesting that the differences in TSH and T4 were secondary to - or at least parallel with - changes in thyroid structure.

A lower prevalence of subclinical hypothyroidism was found among smokers than among non-smokers. The prevalence of subclinical hypothyroidism was $2 \cdot 6 \%(n=41)$ among smokers and $5 \cdot 3 \%(n=147)$ among non-smokers, and OR was 0.47 (95\% CI 0.33-0.67) with adjustment for age, gender and iodine status, when subclinical hypothyroidism was defined as a TSH $>3.6 \mathrm{mU} / \mathrm{l}$. With a limit for TSH of $5.0 \mathrm{mU} / 1$, the OR was similar, but with wider confidence intervals (OR 0.55; CI 0.33-0.92). The difference between smokers and non-smokers was similar for autoimmune and non-autoimmune subclinical hypothyroidism; for autoimmune subclinical hypothyroidism, the prevalence was $1 \cdot 2 \%$ among smokers and $2 \cdot 8 \%$ among non-smokers, thus representing approximately half of the cases of hypothyroidism in both groups. No association was found between smoking and subclinical hyperthyroidism, defined as a serum TSH $<0.4 \mathrm{mU} / 1$ with observed prevalences of $5 \cdot 3 \%(n=85)$ among smokers and $4 \cdot 7 \%(n=129)$ among non-smokers (OR 1.15; CI 0.87-1.53).

\section{Discussion}

We report a positive association between smoking habits and the prevalence of thyroid multinodularity at ultrasonography. No previous studies have focused on this association but, in a Swedish study, the increased prevalence of goitre was almost entirely constituted by multinodular glands (Christensen et al. 1984), and in an Italian study nodular goitre dominated among smokers, but not to the same extent among non-smokers (Lio et al. 1989). The tendency in our study towards a stronger association in the area with the most pronounced iodine deficiency is in line with findings regarding the association between tobacco smoking and goitre or thyroid enlargement, as this association is stronger in iodine-deficient areas (Knudsen et al. 2002). This could support the idea that the effect of smoking on the formation of multiple nodules is mediated through thiocyanate, which is a degradation product of cyanide in tobacco smoke, and thiocyanate has been shown to be the major goitrogen from tobacco smoke (Fukayama et al. 1992). Thus, tobacco smoking may induce intrathyroidal iodine depletion, and iodine deficiency is one of the factors that are thought to facilitate the formation of multinodular glands. However, we found no difference in the occurrence of thyroid multinodularity between our two regions with slightly different iodine intake (Knudsen et al. 2000a).

Another significant observation was that solitary thyroid nodules showed no association with smoking habits. This was surprising, as some of the solitary nodules may represent early stages of multinodular goitres. On the other hand, it is possible that the occurrence of true adenomas in the thyroid is reduced among smokers, as the incidence of thyroid cancer has been found to be lower among smokers (Galanti et al. 1996, Kreiger \& Parkes 2000, Rossing et al. 2000). This could be a parallel with the low incidence of particularly papillary thyroid cancer in iodine-deficient areas. It should be underlined, however, that the solitary nodules in our study most probably entirely represent benign neoplasms. No malignancies have been detected, and the malignancy rate in 'incidentalomas' is generally low (Stark et al. 1983, Brander et al. 1989, Knudsen et al. $2000 c$ ).

The difference in the associations with tobacco smoking between multiple and solitary nodules supports the idea that the distinction between the two groups is important when investigating aetiology and risk factors. Associations with age, gender and alcohol consumption have also been reported to differ between multiple and solitary thyroid nodules (Knudsen et al. 2000c, 2001).

The prevalence of subclinical hypothyroidism was reduced by approximately 50\% among smokers compared with non-smokers in our study, and smokers had significantly lower levels of serum TSH. The association between tobacco smoking and hypothyroidism is 
controversial; previous results are not homogenous as recently reviewed (Vestergaard 2002).

A Danish study from a moderately iodine-deficient region found a significantly increased occurrence of hypothyroidism among smokers (Vestergaard et al. 2002). The discrepancy with our data could in part be caused by the different design of the studies and different selection procedures; the study of Vestergaard et al. (2002) was a case-control study with highly selected patients, as only a part of hypothyroid patients will be referred to hospital care. The self-selection in the control group, where smokers are less prone to participate (Knudsen 2001), tends to bias towards a positive association between smoking and hypothyroidism. Another explanation for the difference could be that we investigated subclinical thyroid disease, whereas Vestergaard et al. (2002) investigated overt and probably often complicated hypothyroidism.

A Swedish, longitudinal study reported a positive association between tobacco smoking and hypothyroidism (Nystrom et al. 1993), but the increased incidence of hypothyroidism in that study was apparently found only among ex-smokers, and a control for age and other possible confounders was not reported. Further, the study was from an iodine-sufficient area. Other studies, also from iodinesufficient areas, have failed to detect an association between smoking and hypothyroidism (Bartalena et al. 1989, Ericsson \& Lindgarde 1991, Prummel \& Wiersinga 1993). However, lower levels of TSH among smokers have generally been reported in most studies (Melander et al. 1981, Eden et al. 1984, Hegedus et al. 1985, Ericsson \& Lindgarde 1991, Petersen et al. 1991, Fisher et al. 1997), and only one study found the reverse association (Sepkovic et al. 1984). Though an iodine dependency of the association between smoking and hypothyroidism is imaginable, no clear picture of this is seen from the available literature.

A possible explanation for an association between tobacco smoking and subclinical hypothyroidism is a protective effect against the inhibitory effect of iodine on the thyroid (Laurberg et al. 1998), a protective effect mediated by the competitive inhibition of iodine uptake by thiocyanate. This effect could be parallel to the observed lower incidences of hypothyroidism with decreasing iodine intake (Knudsen et al. 1999b). It is possible that in our study we observed a lower occurrence of subclinical hypothyroidism among smokers as a direct effect of iodine depletion to the thyroid gland, whereas overt, severe hypothyroidism involves other mechanisms associated with smoking. This is supported by the absent involvement of thyroid autoimmunity in the association between smoking and subclinical hypothyroidism in our study, whereas most cases of overt, spontaneous hypothyroidism in Denmark involve thyroid autoimmunity (Knudsen et al. 1999b). A negative association between thyroglobulin antibodies and tobacco smoking was found in this cohort, whereas the association between TPO antibodies and smoking was weaker (Bulow et al. 2001).
Thus, the transition from subclinical to overt hypothyroidism could be enhanced among smokers, and this could explain the conflicts with particularly the Danish results of Vestergaard et al. (2002). The number of overt, undiagnosed cases of thyroid dysfunction was too small in our study to allow reliable analyses, and including known thyroid disease would have introduced the possibility of considerable selection bias.

We found no association between smoking and subclinical hyperthyroidism, although increased incidence of autoimmune hyperthyroidism has been found among smokers (Bartalena et al. 1989, Prummel \& Wiersinga 1993), and an increased prevalence of thyroid autonomy could have been anticipated along with the increased prevalence of thyroid multinodularity. The increased levels of T4 and low TSH levels among smokers could still suggest a certain level of autonomous hormone secretion from the multiple nodules observed at ultrasonography.

In conclusion, a positive association was found between tobacco smoking and thyroid multinodularity and a negative association was found between smoking and subclinical hypothyroidism in areas with mild to moderate iodine deficiency. An iodine-blocking effect of thiocyanate on the thyroid is a possible explanation for these associations. Other mechanisms may be involved in the observed associations, as other mechanisms must be involved in the association between smoking and Graves' disease and ophthalmopathy.

\section{Acknowledgements}

We express our thanks to Inge-Lise Legaard and Rene Fiege who carefully performed the ultrasonographies. This study was supported by grants from Tømmerhandler Vilhelm Bang Foundation, the Copenhagen Hospital Corporation Research Foundation, the 1991 Pharmacy Foundation, the Danish Medical Foundation, the Health Insurance Foundation and North Jutland County Research Foundation.

\section{References}

Barrere X, Valeix P, Preziosi P, Bensimon N, Pelletier B, Galan P, \& Hercberg S 2000 Determinants of thyroid volume in healthy French adults participating in the SU.VI.MAX cohort. Clinical Endocrinology 52 273-278.

Bartalena L, Martino E, Marcocci C, Bogazzi F, Panicucci M, Velluzzi F, Loviselli A \& Pinchera A 1989 More on smoking habits and Graves' ophthalmopathy. Journal of Endocrinological Investigation $12733-737$.

Bartalena L, Bogazzi F, Tanda ML, Manetti L, Dell'Unto E \& Martino E 1995 Cigarette smoking and the thyroid. European Journal of Endocrinology 133 507-512.

Bertelsen JB \& Hegedus L 1994 Cigarette smoking and the thyroid. Thyroid 4 327-331.

Brander A, Viikinkoski P, Nickels J \& Kivisaari L 1989 Thyroid gland: US screening in middle-aged women with no previous thyroid disease. Radiology 173 507-510. 
Brix TH, Hansen PS, Kyvik KO \& Hegedus L 2000 Cigarette smoking and risk of clinically overt thyroid disease: a population-based twin case-control study. Archives of Internal Medicine 160 661-666.

Bulow I, Knudsen N, Perrild H, Jørgensen T, Ovesen L \& Laurberg P 2001 Association of smoking and the prevalence of TPO-Ab and $\mathrm{Tg}-\mathrm{Ab}$ in a population study. Journal of Endocrinological Investigation 2439 (Abstract).

Christensen SB, Ericsson UB, Janzon L, Tibblin S \& Melander A 1984 Influence of cigarette smoking on goiter formation, thyroglobulin, and thyroid hormone levels in women. Journal of Clinical Endocrinology and Metabolism 58 615-618.

Eden S, Jagenburg R, Lindstedt G, Lundberg PA \& Mellstrom D 1984 Thyroregulatory changes associated with smoking in 70-year-old men. Clinical Endocrinology 21 605-610.

Ericsson UB \& Lindgarde F 1991 Effects of cigarette smoking on thyroid function and the prevalence of goitre, thyrotoxicosis and autoimmune thyroiditis. Journal of Internal Medicine 229 67-71.

Fisher CL, Mannino DM, Herman WH \& Frumkin H 1997 Cigarette smoking and thyroid hormone levels in males. International Journal of Epidemiology 26 972-977.

Fukayama H, Nasu M, Murakami S \& Sugawara M 1992 Examination of antithyroid effects of smoking products in cultured thyroid follicles: only thiocyanate is a potent antithyroid agent. Acta Endocrinologica 127 520-525.

Galanti MR, Hansson L, Lund E, Bergstrom R, Grimelius L, Stalsberg H, Carlsen E, Baron JA, Persson I \& Ekbom A 1996 Reproductive history and cigarette smoking as risk factors for thyroid cancer in women: a population-based case-control study. Cancer, Epidemiology, Biomarkers and Prevention 5 425-431.

Georgiadis E, Papapostolou C, Korakis T, Evagelopoulou K, Mantzoros C \& Batrinos M 1997 The influence of smoking habits on thyroid gland volume: an ultrasonic approach. Journal of the Royal Society of Health 117 355-358.

Hart P, Farrell GC, Cooksley WG \& Powell LW 1976 Enhanced drug metabolism in cigarette smokers. British Medical Journal 2 147-149.

Hegedus L, Karstrup S, Veiergang D, Jacobsen B, Skovsted L \& Feldt-Rasmussen U 1985 High frequency of goitre in cigarette smokers. Clinical Endocrinology 22 287-292.

Knudsen N 2001 Regional variations in goitre prevalence in Denmark - and the impact of tobacco smoking. PhD Thesis. University of Copenhagen. (Available from the author atnk01@bbh.hosp.dk)

Knudsen N, Bols B, Bulow I, Jørgensen T, Perrild H, Ovesen L \& Laurberg P 1999a Validation of ultrasonography of the thyroid gland for epidemiological purposes. Thyroid 9 1069-1074.

Knudsen N, Jørgensen T, Rasmussen S, Christiansen E \& Perrild H $1999 b$ The prevalence of thyroid dysfunction in a population with borderline iodine deficiency. Clinical Endocrinology 51 361-367.

Knudsen N, Bulow I, Jørgensen T, Laurberg P, Ovesen L \& Perrild H 2000a Goitre prevalence and thyroid abnormalities at ultrasonography. A comparative epidemiological study in two regions with slightly different iodine status. Clinical Endocrinology $\mathbf{5 3}$ 479-485.

Knudsen N, Bulow I, Jørgensen T, Laurberg P, Ovesen L \& Perrild H $2000 b$ Comparative study of thyroid function and types of thyroid dysfunction in two areas in Denmark with slightly different iodine status. European Journal of Endocrinology 143 485-491.

Knudsen N, Perrild H, Christiansen E, Rasmussen S, Dige-Petersen H \& Jørgensen T 2000c Thyroid structure and size and two-year follow-up of solitary cold thyroid nodules in an unselected population with borderline iodine deficiency. European Journal of Endocrinology 142 224-230.
Knudsen N, Bulow I, Laurberg P, Perrild H, Ovesen L \& Jørgensen T 2001 Alcohol consumption is associated with reduced prevalence of goitre and solitary thyroid nodules. Clinical Endocrinology $\mathbf{5 5}$ 41-46.

Knudsen N, Bulow I, Laurberg P, Ovesen L, Perrild H \& Jørgensen T 2002 Association of tobacco smoking with goiter in a low-iodine-intake area. Archives of Internal Medicine 162 439-443.

Kreiger N \& Parkes R 2000 Cigarette smoking and the risk of thyroid cancer. European Journal of Cancer 36 1969-1973.

Laurberg P, Pedersen KM, Vestergaard H \& Sigurdsson G 1991 High incidence of multinodular toxic goitre in the elderly population in a low iodine intake area vs high incidence of Graves' disease in the young in a high iodine intake area: comparative surveys of thyrotoxicosis epidemiology in East-Jutland, Denmark and Iceland. Journal of Internal Medicine 229 415-420.

Laurberg P, Pedersen KM, Hreidarsson A, Sigfusson N, Iversen E \& Knudsen PR 1998 Iodine intake and the pattern of thyroid disorders: a comparative epidemiological study of thyroid abnormalities in the elderly in Iceland and in Jutland, Denmark. Journal of Clinical Endocrinology and Metabolism 83 765-769.

Lio S, Napolitano G, Marinuzzi G \& Monaco F 1989 Role of smoking in goiter morphology and thyrotropin response to TRH in untreated goitrous women. Journal of Endocrinological Investigation 12 93-97.

Melander A, Westgren U, Ericson LE \& Sundler F 1977 Influence of the sympathetic nervous system on the secretion and metabolism of thyroid hormone. Endocrinology 101 1228-1237.

Melander A, Nordenskjold E, Lundh B \& Thorell J 1981 Influence of smoking on thyroid activity. Acta Medica Scandinavica 209 41-43.

Nygaard B, Gideon P, Dige-Petersen H, Jespersen N, Solling K \& Veje A 1993 Thyroid volume and morphology and urinary iodine excretion in a Danish municipality. Acta Endocrinologica 129 505-510.

Nystrom E, Bengtsson C, Lapidus L, Petersen K \& Lindstedt G 1993 Smoking - a risk factor for hypothyroidism. Journal of Endocrinological Investigation 16 129-131.

Petersen K, Lindstedt G, Lundberg PA, Bengtsson C, Lapidus L \& Nystrom E 1991 Thyroid disease in middle-aged and elderly Swedish women: thyroid-related hormones, thyroid dysfunction and goitre in relation to age and smoking. Journal of Internal Medicine 229 407-413.

Prummel MF \& Wiersinga WM 1993 Smoking and risk of Graves' disease. Journal of the American Medical Association 269 479-482.

Rossing MA, Cushing KL, Voigt LF, Wicklund KG \& Daling JR 2000 Risk of papillary thyroid cancer in women in relation to smoking and alcohol consumption. Epidemiology 11 49-54.

Sepkovic DW, Haley NJ \& Wynder EL 1984 Thyroid activity in cigarette smokers. Archives of Internal Medicine 144 501-503.

Stark DD, Clark OH, Gooding GA \& Moss AA 1983 High-resolution ultrasonography and computed tomography of thyroid lesions in patients with hyperparathyroidism. Surgery 94 863-868.

Vestergaard P 2002 Smoking and thyroid disorders - a meta-analysis. European Journal of Endocrinology 146 153-161.

Vestergaard P, Rejnmark L, Weeke J, Hoeck HC, Nielsen HK, Rungby J, Laurberg P \& Mosekilde L 2002 Smoking as a risk factor for Graves' disease, toxic nodular goiter, and autoimmune hypothyroidism. Thyroid 12 69-75.

Received 1 July 2002

Accepted 5 September 2002 